Artículo de investigación.

Cómo citar: N. Sánchez, y I. Lira.

"La Manufactura aditiva como potencializador de los sitemas productivos". Inventum, vol. 15, No. 28, pp 2 - 13. DOI: 10.26620/uniminuto. inventum.15.28.2020.104-112.

Editorial: Corporación Universitaria Minuto de Dios - UNIMINUTO.

ISSN: $1909-2520$

eISSN: $2590-8219$

Fecha de recibido: 10 de enero del 2020 Fecha de aprobado: 20 de marzo del 2020 Fecha de publicación: 30 de mayo del 2020

Conflicto de intereses: los autores han declarado que no existen intereses en competencia.

\section{La manufactura aditiva como potenciador de los sistemas productivos}

\author{
Additive manufacturing as a \\ boost of productive systems
}

\author{
Fabricação aditiva como um \\ melhorador para sistemas de produção
}

\begin{abstract}
Resumen
Actualmente, el desarrollo de los nuevos productos necesita de un diseño y manufactura basados en métodos avanzados de producción, y es así como aparece un nuevo enfoque de trabajo conocido como la manufactura aditiva. En este artículo se pretende hacer una revisión de la integración de la manufactura aditiva a las nuevas formas de operación, cuyo objetivo principal es obtener un enfoque preciso en la ingeniería asistida por computadora, con un método del elemento finito y los procesos que conlleva, para así conseguir el producto adecuado y reducir los costos de impresión y tiempo de procesamiento.
\end{abstract}

Para ello se introduce el uso de sistemas CAD/CAE/CAM, que por sus siglas en inglés significan lo siguiente: Computer Aided Design (CAD), Computer Aided Engineering (CAE) Computer Aided Manufacturing (CAM) como herramienta de diseño y manufactura de procesos. La aplicación de estas herramientas está inmersa en la Industria 4.0, y en este documento se muestra lo que implica la generación de modelos en tres dimensiones, así como la realización de pruebas de funcionalidad de prototipos en diferentes situaciones, así como la ayuda en la obtención de resultados confiables en el diseño de cualquier sistema tecnológico e innovador.

Palabras clave: CAD, CAM, CAE, Diseño, Industria 4.0, Manufactura, 3D.

\begin{abstract}
Currently, the development of new products needs to be designed and manufactured with advanced methods to be at the forefront, so a new work approach known as additive manufacturing appears. It is intended in this work a review of the integration of additive manufacturing to new forms of operation, whose main objective is to obtain a precise focus on computer-aided engineering through the finite element method and the processes involved, to thus getting the right product reducing printing costs and processing time.
\end{abstract}

For this purpose, the use of CAD / CAE / CAM systems is introduced, which means the following: Computer Aided Design (CAD), Computer Aided Engineering (CAE) Computer Aided Manufacturing (CAM) as a design and manufacturing tool for processes. The application of these tools is immersed in Industry 4.0, and this document shows how the generation 
of three-dimensional models implies, the testing of functionality of prototypes in different situations, as well as helping to obtain reliable results in the design of any technological and innovative system.

Keywords: CAD, CAM, CAE, Manufacuring, 3D, Design, Industry 4.0

\section{Resumo}

Atualmente, o desenvolvimento de novos produtos requer um projeto $\mathrm{e}$ fabricação com base em métodos de produção avançados e É assim que uma nova abordagem de trabalho conhecida como o manufatura aditiva. Este artigo pretende fazer uma revisão da integração da manufatura aditiva a novas formas de operação, cujo objetivo principal é obter um foco preciso na engenharia auxiliada por computador, com um método de elementos finitos e os processos envolvidos, a fim de obter o produto certo e reduza os custos de impressão e o tempo de processamento. Para isso, é introduzida a utilização de sistemas CAD / CAE / CAM, que devido ao seu representa o seguinte: Design Auxiliado por Computador (CAD), Computer Aided Engineering (CAE) Computer Aided Manufacturing (CAM) como uma ferramenta de projeto e manufatura de processos. A aplicação de essas ferramentas estão imersas na Indústria 4.0 e neste documento Mostra o que implica a geração de modelos em três dimensões, bem como o desempenho de testes de funcionalidade de protótipo em diferentes situações, além de auxiliar na obtenção de resultados confiável no projeto de qualquer sistema tecnológico e inovador.

Palavras-chave: CAD, CAM, CAE, Design, Indústria 4.0, Manufatura, 3D. 


\section{INTRODUCCIÓN}

Hoy en día, la industria necesita adaptarse a las nuevas tecnologías emergentes que existen en el entorno global, esto permite simplificar, optimizar y elevar la calidad de los procesos de diseño y manufactura [1].

El diseño, la ingeniería y la manufactura asistida por computadora (CAD/CAE/CAM) es una disciplina que estudia el uso de sistemas informáticos como herramienta de soporte en todos los procesos involucrados en el diseño, análisis y fabricación de cualquier tipo de producto [2]. Esta disciplina se ha convertido en un recurso sumamente indispensable para la industria, tan revolucionaria e innovadora, en la que vivimos, dicha industria se enfrenta a la necesidad de otorgar calidad en el producto, disminuir los costos y acortar los tiempos de diseño, ingeniería y producción [3]

El término CAX (Computer Aided $\mathrm{X}=$ Technology) hace referencia al uso de diversas tecnologías asistidas por computadora, entre ellas se encuentran las siguientes: CAD, CAE, CAM, CAPP, CAQ. Sin embargo, en este trabajo únicamente consideraremos las tres siguientes: CAD/CAE/CAM [4].

Las herramientas usadas de acceso a la información y conocimiento son el uso de la tecnología por computadora para ayudar al diseño, análisis, planeación de la producción, manufactura de productos, etc. Un sistema CAX puede integrar sistemas de administración, trabajar en conjunto con la automatización para diferentes aspectos del producto, donde generalmente tienen componentes principales como la interacción con las computadoras, software especializado, datos y conocimiento de las actividades del hombre [5].

Actualmente, las empresas requieren diseñar, optimizar, manufacturar y colocar en el mercado productos innovadores y originales para ser competitivas, además se trabaja arduamente para aumentar la participación que se tiene en el mercado o por lo menos para permanecer con la misma proporción.

Por lo tanto, crear rápidamente productos nuevos e innovadores, cuyo rediseño constante permita un mejor desempeño en la función para la cual fue creada, es una alternativa muy eficiente y genera impactos significativos como ventaja frente a sus competidores [6]. El uso cooperativo de estas herramientas permite que las empresas desarrollen productos de gran impacto, sin desperdicios exagerados y con el mejor aprovechamiento de los recursos. Mediante las impresoras 3D, se pueden realizar piezas con materiales revolucionarios en poco tiempo. Se logra una disminución importante en los costos de producción y una mayor rapidez en el diseño de prototipos.

La Industria 4.0 es un paradigma de transformación del sector industrial, permite la unificación entre el mundo físico y el digital, y la conectividad entre los diferentes usuarios. Los países industrializados están adoptando este cambio, desarrollando iniciativas para impulsarlo [7].

De igual manera, la Industria 4.0 abre la industria a nuevas tecnologías que las empresas deben incorporar en sus procesos, como robótica, realidad virtual y aumentada, Internet de las Cosas, etc., y que forman parte de ese conjunto de facilitadores de la transformación digital. La industria médica no puede ser ajena a este cambio; pero necesita estímulos y la confianza de las tecnologías que les son familiares como las herramientas de ayuda al diseño y la construcción (CAD/CAM/CAE), que constituyen la base sobre la que se asientan diversos procesos productivos y de impresión 3D [8], [9].

\section{DESARROLLO DE CONTENIDOS}

El CAD involucra la tecnología con el uso de las computadoras para realizar tareas de creación, modificación, análisis y optimización de un diseño. De esta forma, cualquier aplicación que incluya una interfaz gráfica y realice alguna tarea de ingeniería se considera software de CAD [10]. Las tecnologías de CAD abarcan desde herramientas de modelado geométrico hasta aplicaciones a medida para el análisis u optimización de un producto especifico. El CAE se refiere a las tareas de análisis, evaluación, simulación y optimización desarrolladas a lo largo del ciclo de vida del producto. El termino CAM se puede definir como el uso de herramientas para la planificación, gestión y control de las operaciones de un proceso productivo mediante una interfaz entre un software y los recursos de producción (Fig. 1).

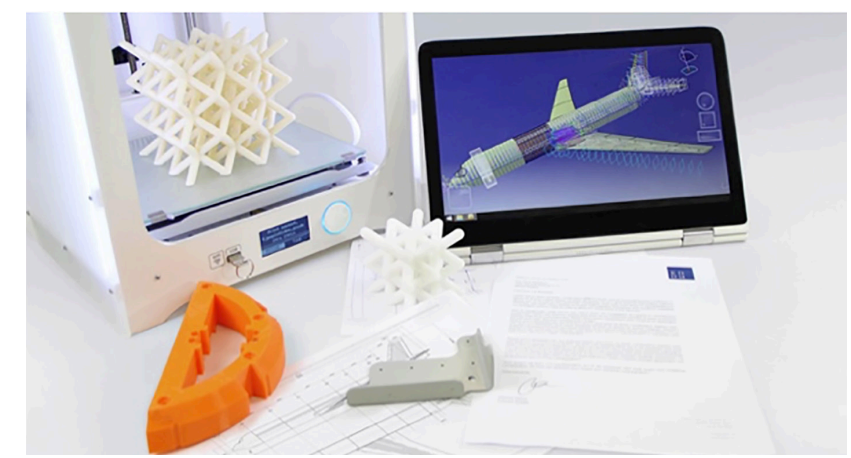

Figura 1. Interfaz CAD y CAM [11].

Fuente: [11]. 
La impresión 3D está abriéndose camino a pasos agigantados en el mundo de la fabricación, sobre todo resulta una muy notable ventaja cuando el producto no se ha empezado a fabricar y se desea obtener una primera imagen real o prototipo, verificar su forma, identificar los pequeños errores que pueden presentarse, en el caso de ser diseñada para mejorar su modelo antecesor, tener la posibilidad de comprobar si el nuevo diseño cumplirá con los requisitos de tamaño y forma en su lugar de trabajo e incluso definir una producción en serie donde al cliente se le da a conocer cómo será su producto antes de comenzar la producción y si este decide hacer una modificación en el diseño, que no suponga una gran pérdida de esfuerzo y dinero [12].

La ventaja principal de la impresión $3 D$ es la flexibilidad a la hora de plantear geometrías de piezas y la posibilidad de fabricar sin útiles, lo que hace que este tipo de fabricación sea totalmente flexible, además de variar geometrías, referencias, combinaciones, etc., lo que brinda una gama de productos más amplia.

La flexibilidad a la hora de generar geometrías que no se podían conseguir antes por métodos de fabricación es una de las características que ha permitido a la manufactura aditiva entrar en el mundo industrial. También en el sector médico, se han encontrado aplicaciones muy prometedoras en prótesis e implantes completamente adaptados a la geometría de los huesos y músculos de los pacientes. También están muy extendidos los útiles y herramientas de fabricación hechos a medida [13].

Los archivos que se generan dentro del CAD/CAE/ CAM y la impresión $3 \mathrm{D}$ tienen vinculación rápida y ágil en los programas que involucran estas tecnologías, en algunos casos se tiene integrado un modulo que permite el enlace entre las aplicaciones, lo cual evidencia la facilidad con la cual se vinculan entre ellas.

\section{METODOLOGÍA}

El diseño asistido por computadora es la tecnología que acude a los sistemas informáticos para asistir a la creación, a la modificación, al análisis, y a la optimización de un diseño. Cualquier programa de computadora que incorpore gráficos y un programa que facilite funciones de ingeniería en los procesos de diseño se puede clasificar como software del CAD.

El papel más básico del CAD es definir la geometría del diseño como una parte mecánica, el ensamble de un producto donde las ventajas más grandes de los sistemas de CAD son: ahorrar tiempo considerable y reducir los errores causados por realizar de otra manera la geometría del diseño y fabricación.

La tecnología CAM involucra sistemas de cómputo que planifican, administran y controlan las operaciones de manufactura mediante un interfaz de cómputo con los recursos de producción de la planta. Una de las áreas más importantes de CAM es el control numérico computarizado (CNC); esta técnica involucra instrucciones programadas para controlar la herramienta de una máquina de corte, o que transforme materia prima en una pieza terminada [14].

Otra función dentro de CAM es la programación de robots, y la planeación de procesos como un objetivo de la automatización por computadora.

La tecnología CAE utiliza un sistema de cómputo para analizar las funciones de un producto creado en CAD, lo que permite a los diseñadores simular y estudiar cómo se comportará el producto, de tal manera que el diseño pueda ser refinado y optimizado. (Fig. 2.) Las herramientas CAE están disponibles para diferentes análisis [15]. 




Figura 2. Herramientas tecnológicas.

Fuente: elaboración propia.

Por ejemplo, los programas de análisis cinemático pueden ser usados para determinar trayectorias de movimiento y velocidades relativas en mecanismo, otro ejemplo son los programas de análisis dinámicos que pueden utilizarse para determinar cargas y desplazamientos en ensambles complejos, como los automóviles [16].

Uno de los métodos más populares es el análisis de elemento finito, este método permite determinar el esfuerzo, la deformación, el calor transferido, la distribución de campo magnético, el flujo de fluidos y otro tipo de problemas que generalmente son difíciles de resolver por otro medio.

\section{CONCLUSIONES}

La impresión 3D, también conocida como manufactura aditiva, es un proceso por el cual se crean objetos físicos, por lo general de material plástico, a partir de capas desde un modelo en $3 \mathrm{D}$ que se encuentra definido en un software. Todos los procesos de impresión 3D requieren que el software, el hardware y los materiales trabajen en conjunto para poder desarrollar el prototipo [17].

La tecnología de impresión 3D puede utilizarse para crear todo tipo de cosas, desde prototipos y piezas simples hasta productos finales altamente técnicos, como piezas para aeronaves, vehículos de transporte, implantes médicos que pueden salvar vidas e incluso órganos artificiales que se producen con capas de células humanas [18].

La posibilidad de imprimir objetos sólidos tridimensionales es algo que actualmente se ha hecho realidad para todas las personas, es una tecnología buscada durante años por diversos fabricantes e investigadores que intentaban implementar un método que permitiera la construcción de los más variados objetos, como implantes médicos, piezas de arquitectura y demás elementos, en forma sencilla y barata [19]. 


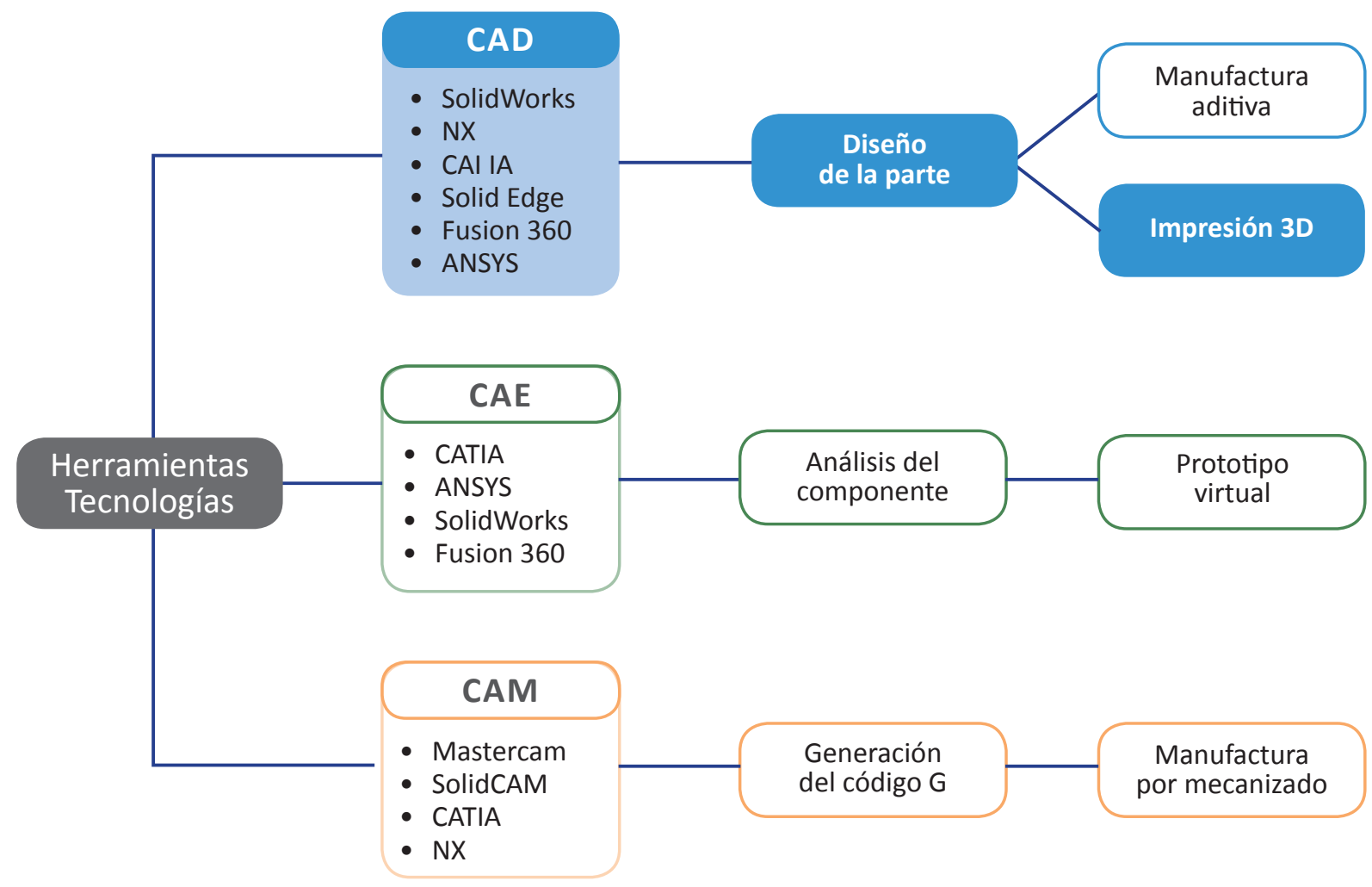

Figura 3. Trayectoria de la investigación posterior.

Fuente: elaboración propia.

En el esquema anterior (Fig. 3.) se muestra la ruta de investigación para un trabajo posterior, en el que se hace uso del CAD con el software CATIA, se genera un diseño de un implante dental y posteriormente se realiza la impresión 3D del mismo.

La competencia es cada día mayor y el tiempo de lanzamiento de cada producto es primordial a la hora de conseguir mayores beneficios. Por último, se puede citar la formación con herramientas CAE de los estudiantes de ingeniería. Uno de los éxitos educacionales consistirá en incluir rotundamente a estos estudiantes en el entorno industrial que le espera, donde los sistemas integrados CAE están convirtiéndose en estándares de la manufactura mundial, se ahorra tiempo y costo [20] (Tabla 1).

La pieza a desarrollar integra un soporte de bisagra automotriz con un espesor de $3 \mathrm{~mm}$ en toda su geometría. 


\begin{tabular}{|c|c|c|c|c|}
\hline Diseño y Desarrollo & $\begin{array}{l}\text { Maquinado de } \\
\text { Prototipos }\end{array}$ & $\begin{array}{l}\text { Producción de } \\
\text { Herramientas }\end{array}$ & Materiales & $\begin{array}{l}\text { Procesamiento } \\
\text { Posterior }\end{array}$ \\
\hline $\begin{array}{l}\text { Diseño } \\
\text { Horas-Hombre } \\
\text { Administración del Proyecto } \\
\text { Simulación de Desempeño } \\
\text { Simulación de Manufactura }\end{array}$ & $\begin{array}{l}1^{\text {a }} \text { Iteración } \\
2^{\text {a }} \text { Iteración } \\
3^{\text {a }} \text { Iteración } \\
4^{\text {a }} \text { Iteración } \\
5^{\text {a Iteración }}\end{array}$ & $\begin{array}{l}1^{\text {a }} \text { Iteración } \\
2^{\text {a }} \text { Iteración } \\
3^{\text {a Iteración }}\end{array}$ & $\begin{array}{l}\text { Volumen de inyección } \\
\text { Tamaño de la prensa } \\
\text { Tiempo de Ciclo } \\
\text { Uso de Planta } \\
\text { Costo y tipo de } \\
\text { Material } \\
\text { Retal de Producción }\end{array}$ & $\begin{array}{l}\text { Tratamiento } \\
\text { Superficial } \\
\text { Guía en el molde } \\
\text { Retrabajo de } \\
\text { producción }\end{array}$ \\
\hline \multicolumn{5}{|l|}{ Ahorro de costos por actividad } \\
\hline $\begin{array}{c}2 \text { días @ } \$ 2.295 \text { por día } \\
\$ 4.950\end{array}$ & $\begin{array}{l}3 \text { Iteraciones @ } \\
\$ 38.250 \\
\text { por Iteración } \\
\$ 114.750\end{array}$ & $\begin{array}{c}1 \text { Iteración @ } \\
\$ 38.250 \\
\text { por Iteración } \\
\$ 38.250\end{array}$ & $\begin{array}{l}10 \% \text { material } \\
20 \% \text { tiempo de ciclo } \\
50 \% \text { retal }\end{array}$ & $\begin{array}{c}1 \text { día @ } 765 \text { por día } \\
\$ 765\end{array}$ \\
\hline \multicolumn{5}{|c|}{ Costo Total Ahorrado: $\$ 158.355$ + ahorro en materiales } \\
\hline \multicolumn{5}{|c|}{ Ahorro de tiempos por actividad } \\
\hline 2 a 3 días & 4 a 6 semanas & 2 a 4 semanas & & 1 a 2 días \\
\hline
\end{tabular}

Tabla 1. Ejemplo de ahorros potenciales en costo y tiempo con el empleo de cae para diseño de una pieza plástica automotriz (en verde - etapas eliminadas al emplear cae, \$ us dollar)

Fuente: [21].

Usar las herramientas de CAD, CAM y CAE, las cuales son muy importantes en desarrollo de algún producto. Estas nos permiten evaluar las características necesarias para el funcionamiento, manufactura, ensamblaje y durabilidad [9].

El sistema de CAE permite obtener información cercana a los esfuerzos que sufriría una pieza, ya que este tipo de análisis numérico es bastante extenso y complejo, además proporciona numerosas ventajas como:

1. Facilidad, comodidad y mayor sencillez en la etapa de diseño.

2. Rapidez, exactitud y uniformidad en la fabricación.

3. Alto porcentaje de éxito.

4. Eliminación de la necesidad de prototipos.

5. Aumento de la productividad.

6. Productos más competitivos.

7. Fácil integración, sin problemas adicionales, en una cadena de fabricación.

8. Se obtiene un producto económico, de óptima calidad y en el menor tiempo posible.
El sistema de CAM permite desarrollar piezas más complejas, debido a que solo se tiene que indicar cuál es el resultado final que se quiere obtener, este tipo de programación es bastante compleja; lo cual lo hace una herramienta muy importante en el desarrollo de moldes [22]. El CAM proporciona una manera rápida de generar el código de forma automática a partir del simple modelo hecho en el primer paso. El código se transfiere a un simple archivo de texto y es introducido en el controlador de la maquinaria de manufactura [23].

Estos sistemas reducen el ciclo de desarrollo, mejoran la calidad y las propiedades deseadas, optimizan los diseños desde el punto de vista estructural, permiten realizar análisis con la utilización de métodos de elementos finitos (esfuerzos, deformaciones, dilataciones térmicas, transferencia de calor, simulación cinemática y dinámica de mecanismos, optimizar los moldes y procesos de fundición o inyección de metales, simulación de inyección de plástico, etc.).

La ventaja principal de la impresión $3 \mathrm{D}$ es la flexibilidad a la hora de plantear geometrías de piezas (que parten de los sistemas CAD), y la posibilidad de fabricar sin modelos, lo que hace que este tipo 
de fabricación se plantee como completamente flexible, por lo tanto, esto facilita la estandarización de modelos 3D, rapidez en la preparación y fabricación en las impresoras, etc.

La manufactura lleva varias décadas siendo aplicada por las empresas y diseñadores; sin embargo, su desarrollo ha sido aislado e independiente. En el presente trabajo se buscó establecer una relación directa entre sus principales aplicaciones y la Industria 4.0, lo que evidencia su importancia para los procesos de fabricación en la actualidad [24].

Las empresas deben adaptar una cultura y procesos a las nuevas necesidades de la sociedad y a las nuevas propuestas empresariales, como lo es en la actualidad la Industria 4.0, ya que una organización con esta nueva visión sin duda generará valor a largo plazo, se reinventará cuando sea necesario y estará mejor preparada para lidiar con los retos económicos, sociales y ambientales que se vislumbran en el nuevo siglo.

\section{REFERENCIAS}

[1] K. Lee, Principles of CAD/CAM/CAE, USA: Addison-Wesley, 1999.

[2] Company, I. H. (1990). "Systems life cycle engineering", Manufacturing Technologies, vol, 13, pp. 83-93.

[3] M. O. Coronado, TRIZ, la metodología más moderna para inventar o innovar tecnológicamente de manera sistemática, México. D.F.: Panorama, 2005.

[4] J. Alcaide Marzal, J. A. Diego Más, y A. Ramírez, Diseño de producto. Métodos y técnicas, Valencia: Universidad Politécnica de Valencia., 2001.

[5] Y. Allen, R. (1996), Materials Processing, Journal of Materials Processing Technology, vol. 13 pp. 181-186.

[6] E. A. Abdulrahman Al-Ahmari, Computer-Aided Inspection Planning, EUA: CRC Press, 2017.

[7] L. G. Antoni Garrell, La Industria 4.0 en la sociedad digital, Barcelona: Marge Books, 2019.
[8] D. A. Barclay I. "Concurrent Engineering: Research and Applications. Management and Organizational Factors in New Product Development (NPD)", Success, vol. 8, $\mathrm{n}^{\circ} .2$, pp. 115-132, 2000.

[9] F. Bianconi, P. Conti, \& L. Di Angelo, "Interoperability among CAD/CAM/CAE systems: a review of current research trends", Geometric Modeling and Imaging--New Trends, vol. 10, $\mathrm{n}^{\circ}$. 11, pp. 82-89, 2004.

[10] F. Bianconi, P. C., "Interoperability among CAD/CAM/CAE Systems: A Review of Current Research Trends", Geometric Modeling and Imaging--New Trends, vol. 10, $\mathrm{n}^{\circ}$. 9, pp. 8289, 2006.

[11] R. P. Fernández y J. Á. Herrero, "La gestión de la Industria 4.0 a través de las herramientas CAD/CAM/CAE", Ingeniería Naval, $\mathrm{n}^{\circ}$. 970, pp. 68-74, 2018.

[12] J. Torres y I. Pastor, "Fabricación avanzada, más allá de la Industria 4.0”, Minsalt, ${ }^{\circ}$. 4, pp. 12-13, 2016.

[13] M. Groover y E. Zimmers, CAD/CAM: Computer-Aided Design and Manufacturing. Prentice Hall International Inc., 2006.

[14] L.L. Delgado, Interacción de sistemas CAD/ CAE en estructuras, tesis de grado, Universidad Autónoma de Chihuahua, México, 1994.

[15] M. Groover y E. Z. CAD/CAM: Computer-Aided Design and Manufacturing, New Jersey: Prentice Hill, 2008.

[16] C. Machover, The CAD/CAM Handbook, USA: McGraw-Hill, 1995.

[17] A., Molina y E. T. "Modelling Manufacturing Capability to Support Concurrent Engineering", Concurrent Engineering: Research and Applications, vol. 3, $\mathrm{n}^{\circ}$. 1, pp. 29-42, 1995.

[18] A. Molina, A.-A., "Research in Engineering Design", A Review of Computer Aided Simultaneous Engineering Systems, vol. 1, $\mathrm{n}^{\circ} .7$, pp. 38-63, 1995.

[19] Moldflow Corporation. (2003). “Cómo predecir problemas de manufactura y optimizar procesos de diseño en el moldeo por inyección?”. [En línea]. Disponible en: 
http://www.plastico.com/temas/Como-predecir-problemas-de-manufactura-y-optimizar-procesos-de-diseno-en-el-moldeo-por-inyeccion +3029748

[20] C. Dym, P. Little, R. Navarra, El proceso de diseño en ingeniería. Cómo desarrollar soluciones efectivas, México: Limusa Wiley, 2002.

[21] O. Putnam, "A redesign for engineering", Harvard Bussiness Review, vol. 01, pp. 139144, 1985.
[22] L. K. Keys, "Design for manufacture design for the life cycle engineering", Fifth IEEE/ CHMT International Electronic Manufacturing Technology Symposium, 1988, 'Design-to-Manufacturing Transfer Cycle, Lake Buena Vista, FL, USA, 1988, pp. 62-72.

[23] R. Udroiu, Computer-aided Technologies. Croatia: IntechOpen, 2016.

[24] I. Zeid, CAD/CAM Theory and Practice. México MacGraw-Hill Inc., 2009. 